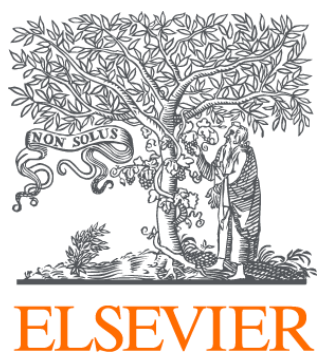

Since January 2020 Elsevier has created a COVID-19 resource centre with free information in English and Mandarin on the novel coronavirus COVID-

19. The COVID-19 resource centre is hosted on Elsevier Connect, the company's public news and information website.

Elsevier hereby grants permission to make all its COVID-19-related research that is available on the COVID-19 resource centre - including this research content - immediately available in PubMed Central and other publicly funded repositories, such as the WHO COVID database with rights for unrestricted research re-use and analyses in any form or by any means with acknowledgement of the original source. These permissions are granted for free by Elsevier for as long as the COVID-19 resource centre remains active. 


\section{Impaired immunogenicity to COVID-19 vaccines in autoimmune systemic diseases. High prevalence of non-response in different patients' subgroups}

Clodoveo Ferri ${ }^{\text {a,b, }}$, Francesco Ursini ${ }^{c}$, Laura Gragnani ${ }^{\mathrm{d}}$, Vincenzo Raimondo ${ }^{\mathrm{b}}$,

Dilia Giuggioli $^{\mathrm{a}}$, Rosario Foti ${ }^{\mathrm{e}}$, Maurizio Caminiti ${ }^{\mathrm{f}}$, Domenico Olivo ${ }^{\mathrm{g}}$, Giovanna Cuomo ${ }^{\mathrm{h}}$, Marcella Visentini ${ }^{\mathrm{i}}$, Fabio Cacciapaglia ${ }^{\mathrm{j}}$, Roberta Pellegrini ${ }^{\mathrm{k}}$, Erika Pigatto ${ }^{1}$, Teresa Urraro ${ }^{\mathrm{m}}$, Caterina Naclerio ${ }^{\mathrm{m}}$, Antonio Tavoni ${ }^{\mathrm{n}}$, Lorenzo Puccetti ${ }^{\mathrm{n}}$, Giuseppe Varcasia ${ }^{\mathrm{o}}$, Ilaria Cavazzana $^{\mathrm{p}}$, Massimo L'Andolina ${ }^{\mathrm{q}}$, Piero Ruscitti ${ }^{\mathrm{r}}$, Marta Vadacca ${ }^{\mathrm{s}}$, Pietro Gigliotti ${ }^{\mathrm{t}}$, Francesca La Gualana ${ }^{i}$, Franco Cozzi ${ }^{1}$, Amelia Spinella ${ }^{a}$, Elisa Visalli ${ }^{e}$, Ylenia Dal Bosco ${ }^{e}$, Giorgio Amato ${ }^{\mathrm{e}}$, Francesco Masini ${ }^{\mathrm{h}}$, Giuseppa Pagano Mariano ${ }^{\mathrm{f}}$, Raffaele Brittelli ${ }^{\mathrm{b}}$, Vincenzo Aiello ${ }^{\mathrm{b}}$, Rodolfo Caminiti ${ }^{\mathrm{b}}$, Daniela Scorpiniti ${ }^{\mathrm{b}}$, Giovanni Rechichi ${ }^{\mathrm{b}}$, Tommaso Ferrari $^{\circ}$, Monica Monti ${ }^{\mathrm{d}}$, Giusy Elia ${ }^{\mathrm{u}}$, Franco Franceschini ${ }^{\mathrm{p}}$, Riccardo Meliconi ${ }^{\mathrm{c}}$, Milvia Casato $^{i}$, Florenzo Iannone ${ }^{j}$, Roberto Giacomelli ${ }^{\mathrm{s}}$, Poupak Fallahi ${ }^{\mathrm{v}}$, Stefano Angelo Santini ${ }^{\mathrm{w}, \mathrm{x}}$, Anna Linda Zignego ${ }^{\mathrm{d}}$, Alessandro Antonelli ${ }^{\mathrm{u}}$

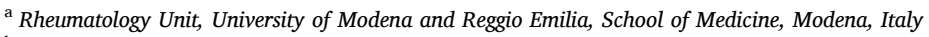

b Rheumatology Clinic 'Madonna Dello Scoglio' Cotronei, Crotone, Italy

${ }^{\mathrm{c}}$ Rheumatology Unit, IRCCS Istituto Ortopedico Rizzoli, Bologna, Italy

${ }^{\mathrm{d}}$ MASVE Interdepartmental Hepatology Center, Department of Experimental and Clinical Medicine, University of Florence Center, Center for Research and Innovation CRIA-MASVE, Firenze, Italy

${ }^{\mathrm{e}}$ Rheumatology Unit AOU Policlinico G. Rodolico - S. Marco, Catania. Italy

${ }^{\mathrm{f}}$ UOD Reumatologia- Grande Ospedale Metropolitano, Reggio Calabria, Italy

${ }^{g}$ Rheumatology Outpatient Clinic, San Giovanni di Dio Hospital, Crotone, Italy

${ }^{\mathrm{h}}$ University of Campania Luigi Vanvitelli, Department of Precision Medicine, Napoli, Italy

i Department of Translational and Precision Medicine, Sapienza University of Rome, Rome, Italy

${ }^{\mathrm{j}}$ UO Reumatologia - DETO, Università di Bari, Bari, Italy

${ }^{\mathrm{k}}$ U.O.C. Medicina Interna "M.Valentini" P.O. Annunziata, Cosenza, Italy

${ }^{1}$ Ospedale "Villa Salus", Mestre, Italy

${ }^{m}$ Rheumatology Unit, "M. Scarlato" Hospital, Scafati, SA, Italy

${ }^{\mathrm{n}}$ Clinical Immunology, University of Pisa, Pisa, Italy

${ }^{\circ}$ U.O.S. Reumatologia, Ospedale Castrovillari, Cosenza, Italy

${ }^{\mathrm{p}}$ Rheumatology, Spedali Civili di Brescia, Brescia, Italy

${ }^{\mathrm{q}}$ Rheumatology Outpatient Clinic, ASP-Vibo Valentia-Tropea Hospital, Italy

${ }^{\mathrm{r}}$ Rheumatology Unit, Department of Biotechnological \& Applied Clinical Sciences, University of L'Aquila, L'Aquila, Italy

${ }^{\mathrm{s}}$ Unità Operativa di Immunoreumatologia-Area Medicina Clinica Policlinico Universitario Campus Bio-Medico di Roma, Roma, Italy

${ }^{\mathrm{t}}$ U.O.T. Specialistica Ambulatoriale, ASP 201, Cosenza, Italy

" Department of Surgical, Medical and Molecular Pathology and Critical Area, University of Pisa, School of Medicine, Pisa, Italy

${ }^{v}$ Department of Translational Research \& New Technologies in Medicine and Surgery, University of Pisa, School of Medicine, Pisa, Italy

${ }^{\mathrm{w}}$ Department of Basic, Clinical, Intensive and Perioperative Biotechnological Sciences, Catholic University School of Medicine, Rome, Italy

${ }^{\mathrm{x}}$ Synlab Italia, Monza, $\mathrm{MB}$, Italy

Abbreviations: Autoimmune systemic diseases, ASD; Rheumatoid factor, RF; Anti-citrullinated protein antibodies, ACPA; Rheumatoid arthritis, RA; Systemic lupus

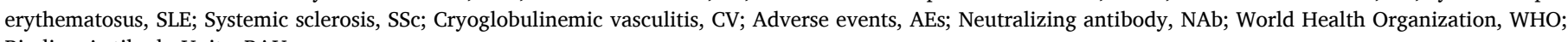
Binding Antibody Units, BAU.

* Corresponding author. Via Aldovrandi 18, S. Giuliano T. Pisa, Italy.

E-mail address: clferri@unimore.it (C. Ferri). 


\section{A R T I C L E I N F O}

\section{Keywords:}

Autoimmune systemic diseases

COVID-19 vaccine

Neutralizing antibodies

Systemic sclerosis

Rheumatoid arthritis

Systemic lupus

Cryoglobulinemic vasculitis

Systemic vasculitis

\begin{abstract}
A B S T R A C T
Autoimmune systemic diseases (ASD) may show impaired immunogenicity to COVID-19 vaccines. Our prospective observational multicenter study aimed to evaluate the seroconversion after the vaccination cycle and at 6-12-month follow-up, as well the safety and efficacy of vaccines in preventing COVID-19.

The study included 478 unselected ASD patients (mean age $59 \pm 15$ years), namely 101 rheumatoid arthritis (RA), 38 systemic lupus erythematosus (SLE), 265 systemic sclerosis (SSc), 61 cryoglobulinemic vasculitis (CV), and a miscellanea of 13 systemic vasculitis. The control group included 502 individuals from the general population (mean age $59 \pm 14$ SD years). The immunogenicity of mRNA COVID-19 vaccines (BNT162b2 and mRNA1273) was evaluated by measuring serum IgG-neutralizing antibody (NAb) (SARS-CoV-2 IgG II Quant antibody test kit; Abbott Laboratories, Chicago, IL) on samples obtained within 3 weeks after vaccination cycle.

The short-term results of our prospective study revealed significantly lower NAb levels in ASD series compared to controls [286 (53-1203) vs 825 (451-1542) BAU/mL, p < 0.0001], as well as between single ASD subgroups and controls. More interestingly, higher percentage of non-responders to vaccine was recorded in ASD patients compared to controls [13.2\% (63/478), vs 2.8\% (14/502); $\mathrm{p}<0.0001$ ]. Increased prevalence of non-response to vaccine was also observed in different ASD subgroups, in patients with ASD-related interstitial lung disease ( $\mathrm{p}=$ $0.009)$, and in those treated with glucocorticoids $(\mathrm{p}=0.002)$, mycophenolate-mofetil $(\mathrm{p}<0.0001)$, or rituximab $(\mathrm{p}<0.0001)$. Comparable percentages of vaccine-related adverse effects were recorded among responder and non-responder ASD patients.

Patients with weak/absent seroconversion, believed to be immune to SARS-CoV-2 infection, are at high risk to develop COVID-19. Early determination of serum NAb after vaccination cycle may allow to identify three main groups of ASD patients: responders, subjects with suboptimal response, non-responders. Patients with suboptimal response should be prioritized for a booster-dose of vaccine, while a different type of vaccine could be administered to non-responder individuals.
\end{abstract}

\section{Introduction}

Observational cohorts demonstrated that patients with autoimmune systemic diseases (ASD) have a higher prevalence of COVID-19 compared to the general population [1-5]. Moreover, worse clinical outcomes, including higher rates of hospitalization and death, have been reported in older ASD patients and/or in those with pre-existing ASD-related lung fibrosis [2,6-8]. Therefore, ASD are now considered among patients' populations highly vulnerable to SARS-CoV-2 infection and morbidity possibly due to their deep immune-system alterations with the possible contribution of ongoing immunosuppressive treatments [2,9-11]. In the early stages of the COVID-19 pandemic, the risk of developing COVID-19 in these patients has been minimized by the stringent lockdown measures adopted by national health authorities together with the broad use of telemedicine allowing the essential contact between patients and referral centers. Subsequently, with the introduction of the COVID-19 mass vaccination campaign, ASD patients were included among population subgroups prioritized for vaccination in several countries, including Italy (https://www.trovanorme.salute. gov.it/norme/renderPdf.spring? seriegu $=$ SG\&datagu $=24 / 03 / 2021 \&$ re $\mathrm{daz}=21 \mathrm{~A} 01802 \&$ artp $=1 \&$ art $=1 \&$ subart $=1 \&$ subart $1=10 \&$ ve

$r s=1 \& \operatorname{prog}=002)$ [12]. Data from clinical trials and real-life studies confirmed a good immunogenicity and safety of available vaccines in ASD patients, in particular those mRNA-based [13-16]. However, a number of factors including immune-system disorders, underlying chronic inflammatory diseases, disease activity at the time of vaccination, concurrent immune-modifier therapies (high glucocorticoid dosages, rituximab, etc.), and older age have been suggested to negatively affect the immune response to vaccines in individual patients and/or particular disease subsets [14-17]. Starting from the last February 2021 the COVID-19 \& Italian ASD Study Group organized a multicenter prospective observational study with the main purpose of evaluating the immunogenicity and safety of COVID-19 vaccination in ASD patients, the possible effects of ongoing treatments, as well as its ability to prevent symptomatic SARS-CoV-2 infection over long-term clinical follow-up. Here we report the data regarding the COVID-19 vaccine immunogenicity and safety evaluated in a large ASD patients' series after the completion of vaccination cycle.

\section{Patients and methods}

Our prospective, long-term, observational, multicenter survey, involved 21 centers of the COVID-19 \& ASD Italian Study Group equally distributed among the Italian regions. The study included some welldefined ASD in order to deal with sufficiently homogeneous and representative disease subgroups. In particular, we consecutively recruited patients with rheumatoid factor (RF) and/or anti-citrullinated protein antibodies (ACPA) positive rheumatoid arthritis (RA), systemic lupus erythematosus (SLE), systemic sclerosis (SSc), cryoglobulinemic vasculitis (CV), and a miscellanea of other systemic vasculitis; disease definition and patients' clinical assessment were carried out following updated classification criteria and current methodologies [18]. The primary aim of the study was the evaluation of the immunogenicity of COVID-19 vaccines at the end of vaccination cycle; secondary aims were to investigate the safety of COVID-19 vaccines and the impact of ongoing immune-modifier medications. The serum samples were collected between March and July 2021 in order to detect the early antibody response (1-3 weeks after the administration of the second dose of vaccine).

Demographic characteristics, specific vaccine administered and vaccine-related adverse events (AEs), measures of disease activity, ASDrelated complications, and ongoing treatment at the time of vaccination were recorded. Regarding the latter, the peri-vaccination management of immune-modifier medications followed the recommendations of the Italian Society of Rheumatology (https://www.reumatologia.it/vacc inazioni), taking in account both individual patient's clinical characteristics (e.g. major comorbidities) and disease activity. Individuals treated with rituximab or cyclophosphamide during the last 6 months before vaccination $(n=7)$ were excluded from the study. Additional exclusion criteria were pregnancy, history of allergic reactions after previous vaccination, and/or recent SARS-CoV-2 infection.

The monitoring of safety in all participants was carried out by telephone interview 1-3 weeks before the first dose, then 2 weeks after the first and second dose in order to detect possible vaccine-related AEs using a standardized symptoms assessment questionnaire. The immunogenicity of COVID-19 vaccines is evaluated by measuring the titer of IgG neutralizing antibody (NAb) against SARS-CoV-2 trimeric spike S1/ S2 glycoproteins on serum samples obtained within 1-3 weeks after completion of the vaccination cycle using the SARS-CoV-2 IgG II Quant 
antibody test kit (Abbott Laboratories, Chicago, IL). As recommended by World Health Organization (WHO), antibody titers are expressed as Binding Antibody Units (BAU)/ml, with a cut-off for positive testing of 7 $\mathrm{BAU} / \mathrm{ml}$.
The study protocol was approved by local ethic committee (RETROCoV2 study code \#17886_bio); informed consent was obtained from all patients before participation.

A sample of 502 individuals ( $73 \%$ females, mean age $59 \pm 14$ years)

Table 1

Comparison between SARS-CoV-2 IgG neutralizing antibodies (NAb) negative and positive individuals.

\begin{tabular}{|c|c|c|c|c|}
\hline & $\begin{array}{l}\text { ASD } \\
\text { Whole series }(n=478)\end{array}$ & $\begin{array}{l}\text { SARS-CoV-2 IgG NAb negative }(\mathrm{n}= \\
63 \text { ) }\end{array}$ & SARS-CoV-2 IgG NAb positive $(n=415)$ & $\begin{array}{l}\text { P value } \\
\text { Odds Ratio } \\
\text { C.I. }\end{array}$ \\
\hline Age, years & $59 \pm 15$ & $61 \pm 15$ & $59 \pm 16$ & 0.284 \\
\hline Female gender, n (\%) & $401(83.9)$ & $50(79.4)$ & $351(84.6)$ & 0.294 \\
\hline Past COVID-19, n (\%) & $15(3.1)$ & $0(0)$ & $15(3.6)$ & 0.125 \\
\hline \multicolumn{5}{|l|}{ ASD-related complications } \\
\hline Interstitial lung disease, $\mathrm{n}(\%)$ & $112(23.4)$ & $23(36.5)$ & $89(21.4)$ & $\begin{array}{l}0.009 \\
2.106(1.198-3.702)\end{array}$ \\
\hline Skin ulcers, $\mathbf{n}(\%)$ & $88(18.4)$ & $12(19.0)$ & $76(18.3)$ & $\begin{array}{l}0.889 \\
1.050(0.534-2.064)\end{array}$ \\
\hline Renal involvement, n (\%) & $18(3.8)$ & $4(6.3)$ & $14(3.4)$ & $\begin{array}{l}0.248 \\
1.942(0.618-6.098)\end{array}$ \\
\hline Pulmonary arterial hypertension, n (\%) & $19(4.0)$ & $5(7.9)$ & $14(3.4)$ & $\begin{array}{l}0.084 \\
2.469(0.858-7.110)\end{array}$ \\
\hline Gastrointestinal involvement, n (\%) & $19(4.0)$ & $2(3.2)$ & $17(4.1)$ & $\begin{array}{l}0.727 \\
0.768(0.173-3.405)\end{array}$ \\
\hline \multicolumn{5}{|l|}{ Treatment } \\
\hline Glucocorticoids, n (\%) & $204(42.7)$ & $38(60.3)$ & $166(40.0)$ & $\begin{array}{l}0.002 \\
2.280(1.327-3.919)\end{array}$ \\
\hline Methotrexate, n (\%) & $78(16.3)$ & $9(14.3)$ & $69(16.6)$ & $\begin{array}{l}0.639 \\
0.836(0.394-1.772)\end{array}$ \\
\hline Sulfasalazine, n (\%) & $4(0.8)$ & $1(1.6)$ & $3(0.7)$ & $\begin{array}{l}0.483 \\
2.215 \\
(0.227-21.631)\end{array}$ \\
\hline Hydroxychloroquine, n (\%) & $112(23.4)$ & $9(14.3)$ & $103(24.8)$ & $\begin{array}{l}0.066 \\
0.505(0.241-1.058)\end{array}$ \\
\hline Leflunomide, n (\%) & $4(0.8)$ & $0(0)$ & $4(1.0)$ & 0.434 \\
\hline Mycophenolate, n (\%) & 79 (16.5) & $21(33.3)$ & $58(14.0)$ & $\begin{array}{l}<0.0001 \\
3.078(1.701-5.567)\end{array}$ \\
\hline Azathioprine, n (\%) & $22(4.6)$ & $1(1.6)$ & $21(5.1)$ & $\begin{array}{l}0.220 \\
0.303(0.040-2.290)\end{array}$ \\
\hline TNF inhibitors, n (\%) & $23(4.8)$ & $1(1.6)$ & $22(5.3)$ & $\begin{array}{l}0.199 \\
0.288(0.038-2.176)\end{array}$ \\
\hline Abatacept, n (\%) & $11(2.3)$ & $1(1.6)$ & $10(2.4)$ & $\begin{array}{l}0.685 \\
0.653(0.082-5.192)\end{array}$ \\
\hline Rituximab, n (\%) & $26(5.4)$ & $12(19.0)$ & $14(3.4)$ & $\begin{array}{l}<0.0001 \\
6.739 \\
(2.956-15.367)\end{array}$ \\
\hline IL-6 inhibitors, n (\%) & $21(4.4)$ & $1(1.6)$ & $20(4.8)$ & $\begin{array}{l}0.243 \\
0.319(0.042-2.416)\end{array}$ \\
\hline JAK inhibitors, n (\%) & $14(2.9)$ & $3(4.8)$ & $11(2.7)$ & $\begin{array}{l}0.354 \\
1.836(0.498-6.773)\end{array}$ \\
\hline Belimumab, n (\%) & $14(2.9)$ & $2(3.2)$ & $12(2.9)$ & $\begin{array}{l}0.901 \\
1.101(0.241-5.039)\end{array}$ \\
\hline \multicolumn{5}{|l|}{ Vaccines administered } \\
\hline BNT162b2, n (\%) & $448(93.7)$ & $60(95.2)$ & $388(93.5)$ & $\begin{array}{l}0.595 \\
1.392(0.409-4.730)\end{array}$ \\
\hline mRNA-1273, n (\%) & $30(6.3)$ & $3(4.8)$ & $27(6.5)$ & $\begin{array}{l}0.595 \\
0.719(0.211-2.442)\end{array}$ \\
\hline \multicolumn{5}{|l|}{ Vaccine-related adverse events } \\
\hline No adverse events, n (\%) & $221(46.2)$ & $29(46.0)$ & $192(46.3)$ & $\begin{array}{l}0.972 \\
0.991(0.582-1.686)\end{array}$ \\
\hline Pain at the injection site, $\mathrm{n}(\%)$ & $182(38.1)$ & $22(34.9)$ & $160(38.6)$ & $\begin{array}{l}0.580 \\
0.855(0.491-1.489)\end{array}$ \\
\hline Fever, n (\%) & $42(8.8)$ & $3(4.8)$ & $39(9.4)$ & $\begin{array}{l}0.226 \\
0.482(0.144-1.609)\end{array}$ \\
\hline Headache, n (\%) & $30(6.3)$ & $5(7.9)$ & $25(6.0)$ & $\begin{array}{l}0.560 \\
1.345(0.495-3.652)\end{array}$ \\
\hline Fatigue, n (\%) & $36(7.5)$ & $8(12.7)$ & $28(6.7)$ & $\begin{array}{l}0.095 \\
2.010(0.872-4.633)\end{array}$ \\
\hline ASD flares, n (\%) & $10(2.1)$ & $2(3.2)$ & $8(1.9)$ & $\begin{array}{l}0.519 \\
1.668(0.346-8.039)\end{array}$ \\
\hline SARS-CoV-2 IgG NAb titre, BAU/mL & $825(451-1542)$ & $0.6(0.2-3.3)$ & $446.5(114.5-1360.1)$ & $<0.0001$ \\
\hline
\end{tabular}

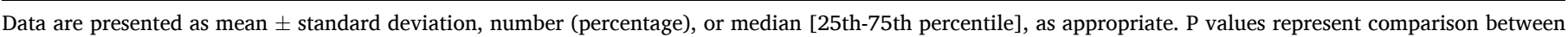

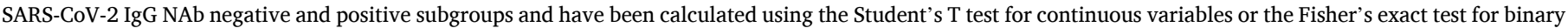
variables.

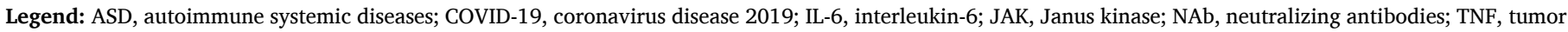
necrosis factor. 
from the general population was used as control group. The same exclusion criteria were applied to the control group; moreover, subjects with a past diagnosis of any ASD and/or a past exposure for any reason to immunosuppressive treatments were excluded $(n=6)$.

Vaccine was administered to patients and controls by intramuscular injection in the deltoid muscle according to the manufacturer indications and Italian national guidelines; the BNT162b2 and mRNA1273 vaccines were administered in 448 (93.7\%) and 30 (6.3\%) ASD patients, respectively, as well as in comparable percentages of control individuals.

\subsection{Statistical analysis}

Data are expressed as mean \pm standard deviation (SD), median (25th - 75th percentile) or number (percentage) as appropriate. The Student's $\mathrm{T}$ test was used for comparing means of continuous variables between two groups; highly skewed variables were ln-transformed before the analysis. Fisher's exact test was used to compare categorical variables. The one-way analysis of variance (ANOVA) and the Kruskal-Wallis $\mathrm{H}$ test were used for comparing three or more groups, as appropriate. Univariate and multivariate logistic regression models were built to assess the predictivity of continuous or categorical variables for a dichotomic dependent variable, expressed as odds ratio (OR) and 95\% confidence interval $(95 \% \mathrm{CI})$. All tests were two tailed. Analyses were performed using the Statistic Package for Social Sciences (SPSS) ${ }^{\circledR}$ software, Version 23 (IBM).

\section{Results}

A total of 478 patients ( $84 \%$ females, mean age $59 \pm 15$ years) were consecutively recruited by the participating centers, including patients with RF and/or ACPA positive RA $(\mathrm{n}=101)$, SLE $(\mathrm{n}=38)$, SSc $(\mathrm{n}=$ $265), \mathrm{CV}(\mathrm{n}=61)$, and a miscellanea of other systemic vasculitis ( $\mathrm{n}=$ 13).

General features of the study population are reported in Table 1 . The serum titer of anti-SARS-CoV-2 IgG NAb, evaluated at the end of the vaccination cycle, was significantly lower in the whole ASD patients series compared to control group [286 (53-1203) vs 825 (451-1542) $\mathrm{BAU} / \mathrm{mL}, \mathrm{p}<0.0001]$; comparable differences were also detected between individual ASD patients' subgroups and controls (Fig. 1A). In addition, the percentage of vaccine non-responders (defined as antiSARS-CoV-2 IgG Nab below threshold) was significantly higher in the whole ASD patients' series than controls [13.2\% (63/478), vs 2.8\% (14/ 502); $\mathrm{p}<0.0001$; Fig. 1B]. Consistently, all ASD patient subgroups showed significantly higher percentages of non-responders compared to controls (Fig. 1B); more evident for both CV and other systemic vasculitis when compared to other subgroups, namely RA, SLE, and SSc patients. (Fig. 1B).

The comparison between anti-SARS-CoV-2 IgG NAb negative $(\mathrm{n}=$ $63)$ and positive ASD $(n=415)$ revealed no differences in gender and age distribution; on the other hand, the number of non-responders was significantly higher in patients with concurrent ASD-related interstitial lung disease (Table 1). Moreover, anti-SARS-CoV-2 IgG NAb negative ASD patients were more frequently treated with glucocorticoids, mycophenolate mofetil, and/or rituximab. Comparable percentages of vaccine-related AEs were recorded among responder and non-responder ASD patients (Table 1).

The comparison between ASD subgroups (Table 2) demonstrated a number of differences at least in part correlated to demographic and clinical features specific for each disorder; namely, an increased prevalence of interstitial lung disease and skin ulcers in SSc patients, as well as of renal involvement in SLE and CV patients. Similarly, differences were also observed as regards the therapeutic regimens; namely, methotrexate and TNF inhibitors were more frequently employed in RA patients, steroids, hydroxychloroquine, and belimumab in SLE, mycophenolate mofetil in SSc, and rituximab in $\mathrm{CV}$ and other systemic vasculitis (Table 2). Of note, none of the patients discontinued immunemodifier medications during the vaccination cycle.

With regard to the safety of vaccine, transitory, mild side effects following the first or second dose of vaccine were recorded in about half of the ASD patients and controls, more frequently headache in SLE and $\mathrm{CV}$ and pain at injection site in systemic vasculitis patients (Table 2).

In addition, disease flares after completion of the vaccination cycle were reported by $10 / 478(2.1 \%)$ ASD patients. Flares included the recurrence of purpura in $6 \mathrm{CV}$ patients, and of skin ulcers in $1 \mathrm{SSc}$ subject, the reactivation of arthritis in a woman with RA, and de novo onset paresthesia in 2 SLE patients.

The possible correlation between impaired vaccine immunogenicity and ongoing immune-modifier treatments was also investigated. A total of 38/63 SARS-CoV-2 IgG NAb negative patients (60.3\%) were receiving glucocorticoids compared to 166/415 (40.0\%) in SARS-CoV-2 IgG NAb positive $(\mathrm{p}=0.002)$. Of note, only $4(6.3 \%)$ and $10(2.4 \%)$ patients were receiving a dose of glucocorticoids greater than $5 \mathrm{mg}$ prednisone equivalent, respectively. Moreover, the percentage of patients treated

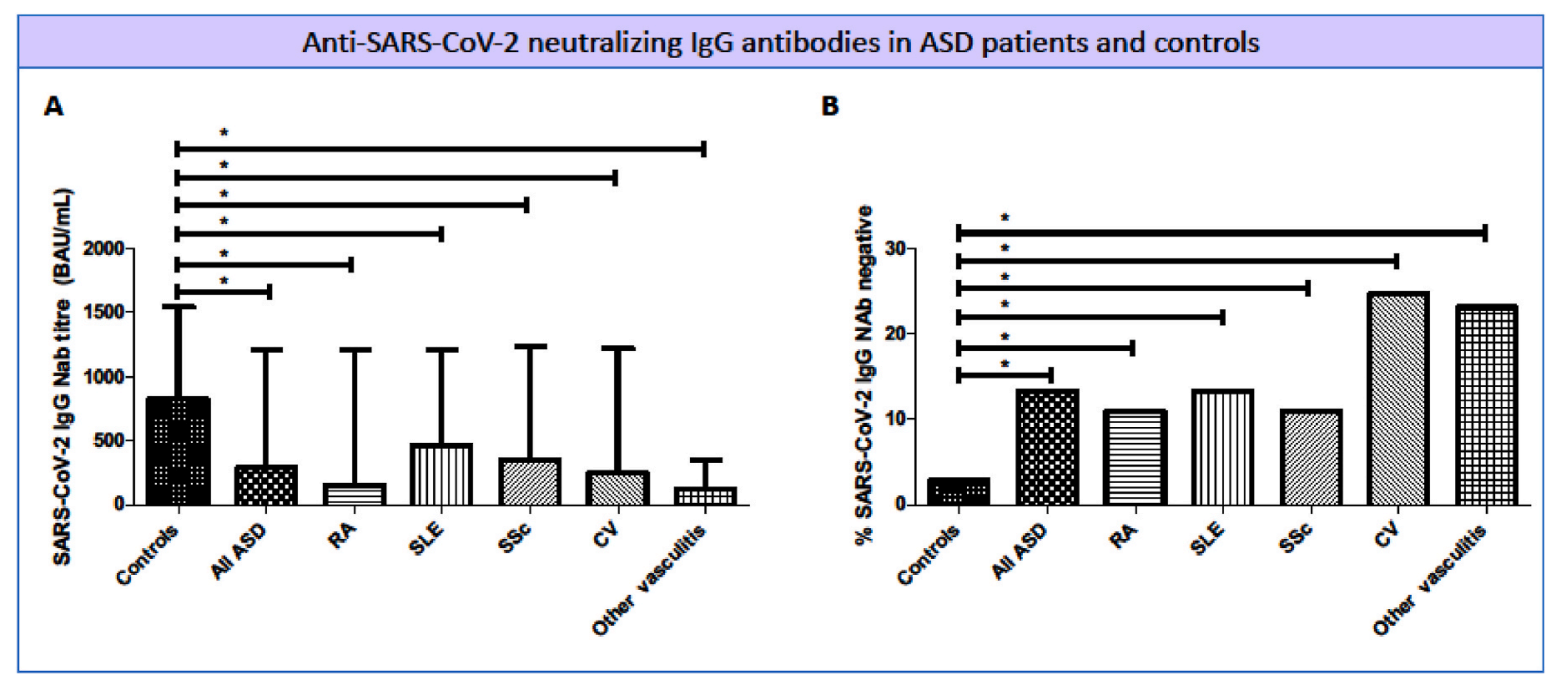

Fig. 1. Anti-SARS-CoV-2 IgG neutralizing antibodies (NAb) titer (A) and percentage of non-responders (B) in autoimmune systemic diseases (ASD) and controls. Anti-SARS-CoV-2 IgG NAb were measured 1-3 weeks after the second dose of vaccine in ASD patients and controls. A statistically significant difference (* indicates $\mathrm{p}<0.05$ ) was between each individual ASD and controls for both NAb titer (panel A) and percentage of non-responders (panel B). Legend: BAU, binding antibody units; CV, cryoglobulinemic vasculitis; RA, rheumatoid arthritis; SLE, systemic lupus erythematosus; SSc, systemic sclerosis. 
Table 2

Analysis of the cohort after stratification for specific autoimmune systemic disease.

\begin{tabular}{|c|c|c|c|c|c|c|}
\hline & $\begin{array}{l}\mathrm{RA}(\mathrm{n}=101) \\
\text { (a) }\end{array}$ & $\begin{array}{l}\text { SLE }(\mathrm{n}=38) \\
\text { (b) }\end{array}$ & $\begin{array}{l}\text { SSc }(n=265) \\
\text { (c) }\end{array}$ & $\begin{array}{l}\mathrm{CV}(\mathrm{n}=61) \\
\text { (d) }\end{array}$ & $\begin{array}{l}\text { Other vasculitis }(\mathrm{n}= \\
\text { 13) } \\
\text { (e) }\end{array}$ & $P$ value \\
\hline Age, years & $61 \pm 12_{\mathrm{b}, \mathrm{d}}$ & $46 \pm 17_{\mathrm{a}, \mathrm{c}, \mathrm{d}}$ & $57 \pm 15_{\mathrm{b}, \mathrm{d}}$ & $74 \pm 11_{\mathrm{a}, \mathrm{b}, \mathrm{c}, \mathrm{e}}$ & $51 \pm 18_{\mathrm{d}}$ & $<0.0001$ \\
\hline Female gender, n (\%) & $71(70.3)$ & $36(94.7)_{a}, \mathrm{e}$ & $236(89.1)_{\mathrm{a}, \mathrm{e}}$ & $51(83.6)$ & $7(53.8)$ & $<0.0001$ \\
\hline Disease duration, years & $8 \pm 7 b$ & $14 \pm 12_{\mathrm{a}, \mathrm{d}}$ & $10 \pm 8$ & $9 \pm 6_{b}$ & $4 \pm 5$ & 0.008 \\
\hline Past COVID-19, n (\%) & $5(5.0)$ & $3(7.9)$ & $7(2.6)$ & $0(0)$ & $0(0)$ & 0.163 \\
\hline \multicolumn{7}{|l|}{ ASD-related complications } \\
\hline Interstitial lung disease, n (\%) & $2(2.0)$ & $1(2.6)$ & $107(40.4)_{a}, \mathrm{~b}$ & $0(0)$ & $2(15.4)$ & $<0.0001$ \\
\hline Skin ulcers, n (\%) & $0(0)$ & $4(10.5)$ & $78(29.4)_{d}$ & $5(8.2)$ & $1(7.7)$ & $<0.0001$ \\
\hline Renal involvement, n (\%) & $0(0)$ & $6(15.8)_{c}$ & $4(1.5)$ & $8(13.1)_{c}$ & $0(0)$ & $<0.0001$ \\
\hline Pulmonary art. hypert., n (\%) & $0(0)$ & $1(2.6)$ & $18(6.8)$ & $0(0)$ & $0(0)$ & 0.012 \\
\hline Gastrointestinal inv., n (\%) & $0(0)$ & $0(0)$ & $19(7.2)$ & $0(0)$ & $0(0)$ & 0.003 \\
\hline \multicolumn{7}{|l|}{ Treatment } \\
\hline Glucocorticoids, n (\%) & $44(43.6)$ & $31(81.6)_{\mathrm{a}}$ & $105(39.8)$ & $16(26.2)_{\mathrm{a}}$ & $8(61.5)$ & $<0.0001$ \\
\hline Methotrexate, n (\%) & $57(56.4)_{b, c}$ & $3(7.9)$ & $15(5.7)$ & $0(0)$ & $3(23.1)$ & $<0.0001$ \\
\hline Sulfasalazine, n (\%) & $4(4.0)$ & $0(0)$ & $0(0)$ & $0(0)$ & $0(0)$ & 0.005 \\
\hline Hydroxychloroquine, n (\%) & $10(9.9)$ & $25(65.8)_{a}, c, d, e$ & $75(28.3)$ a, d & $1(1.6)$ & $1(7.7)$ & $<0.0001$ \\
\hline Leflunomide, $\mathrm{n}(\%)$ & $2(2.0)$ & $1(2.6)$ & $1(0.4)$ & $0(0)$ & $0(0)$ & 0.359 \\
\hline Mycophenolate, n (\%) & $0(0)$ & $4(10.5)$ & $73(27.5)_{a}$ & $0(0)$ & $2(15.4)$ & $<0.0001$ \\
\hline Azathioprine, n (\%) & $0(0)$ & $4(10.5)$ & $15(5.7)$ & $0(0)$ & $3(23.1)_{c}$ & $<0.0001$ \\
\hline TNF inhibitors, n (\%) & $21(20.8)_{c}$ & $0(0)$ & $1(0.4)$ & $0(0.0)$ & $1(7.7)_{c}$ & $<0.0001$ \\
\hline Abatacept, n (\%) & $6(5.9)_{c}$ & $0(0)$ & $5(1.9)$ & $0(0)$ & $0(0)$ & 0.067 \\
\hline Rituximab, n (\%) & $2(2.0)$ & $0(0)$ & $14(5.3)$ & $8(13.1)_{a}$ & $2(15.4)$ & 0.007 \\
\hline IL-6 inhibitors, n (\%) & $6(5.9)$ & $0(0)$ & $13(4.9)$ & $0(0)$ & $2(15.4)$ & 0.060 \\
\hline JAK inhibitors, n (\%) & $14(13.9)$ & $0(0)$ & $0(0)$ & $0(0)$ & $0(0)$ & $<0.0001$ \\
\hline Belimumab, n (\%) & $0(0)$ & $14(36.8)$ & $0(0)$ & $0(0)$ & $0(0)$ & $<0.0001$ \\
\hline \multicolumn{7}{|l|}{ Vaccines administered } \\
\hline BNT162b2, n (\%) & $93(92.1)$ & $34(89.5)$ & 253 (95.5) & $55(90.2)$ & $13(100)$ & 0.269 \\
\hline mRNA-1273, n (\%) & $8(7.9)$ & $4(10.5)$ & $12(4.5)$ & $6(9.8)$ & $0(0)$ & 0.268 \\
\hline \multicolumn{7}{|l|}{ Vaccine-related adverse events } \\
\hline No adverse events, n (\%) & $56(55.4)_{\mathrm{d}}$ & $21(55.3)_{\mathrm{d}}$ & $125(47.2)_{\mathrm{d}}$ & $15(24.6)$ & $4(30.8)$ & 0.002 \\
\hline Pain at the injection site, $n(\%)$ & $33(32.7)$ & $15(39.5)$ & $96(36.2)$ & $28(45.9)$ & $10(76.9)_{\mathrm{a}, \mathrm{c}}$ & 0.021 \\
\hline Fever, n (\%) & $8(7.9)$ & $5(13.2)$ & $22(8.3)$ & $4(6.6)$ & $3(23.1)$ & 0.312 \\
\hline Headache, n (\%) & $9(8.9)$ & $6(15.8)_{c}$ & $8(3.0)$ & $7(11.5)_{c}$ & $0(0)$ & 0.004 \\
\hline Fatigue, n (\%) & $5(5.0)$ & $1(2.6)$ & $28(10.6)$ & $2(3.3)$ & $0(0)$ & 0.077 \\
\hline SARS-CoV-2 IgG NAb titre, BAU/ & 153.05 & 455.87 & 349.48 & 245.08 & 118.83 & 0.184 \\
\hline $\mathrm{mL}$ & [52.72-1140.23] & [129.16-1028.31] & {$[63.21-1224.2]$} & [7.33-1072.00] & {$[46.92-317.90]$} & \\
\hline Vaccine non-response, n (\%) & $11(10.9)$ & $5(13.2)$ & $29(10.9)$ & $15(24.6)$ a, b, c & $3(23.1)_{a}, b, c$ & 0.047 \\
\hline
\end{tabular}

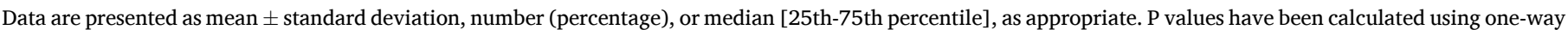

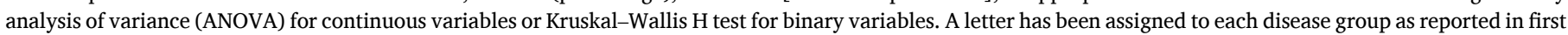

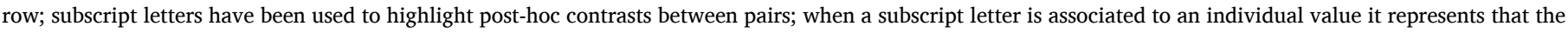
value differs from that observed in the group associated with the letter.

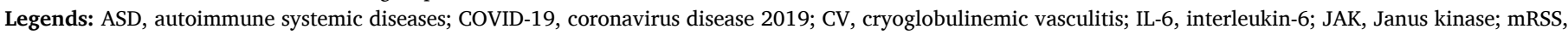

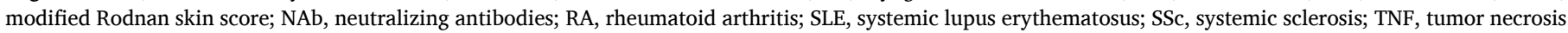
factor.

with mycophenolate mofetil ( $\mathrm{p}<0.0001$ ) or rituximab ( $\mathrm{p}<0.0001$ ) was significantly higher in non-responders compared to the responder subgroup.

Univariate logistic regression analysis was carried out to investigate possible predictors of vaccine non-response in ASD patients. Pre-existent ASD-related interstitial lung disease (OR: 2.106, 95\% CI: 1.198-3.702, p $=0.010$ ), treatment with glucocorticoids (OR: 2.27, 95\% CI: 1.321-3.903, $\mathrm{p}=0.003$ ), mycophenolate mofetil (OR: 3.078, 95\% CI: 1.701-5.567, $\mathrm{p}<0.0001$ ) or rituximab (OR: 6.739, 95\% CI: 2.956-15.367, $\mathrm{p}<0.0001$ ) were associated with higher odds of vaccine non-response (Table 3). In a multivariate logistic regression model including only variables that yielded significant association with vaccine non-response in univariate analysis to avoid model overfitting, only treatment with rituximab maintained its predictivity in impairing the immunogenicity of anti-SARS-CoV-2 vaccine (OR: $4.031,95 \%$ CI: 1.464-11.105, $\mathrm{p}=0.007$ ).

Finally, the ASD responders and non-responders' subgroups did not show significant correlations with the type of vaccine administered or with the prevalence of vaccine-related side effects (Table 1).

\section{Discussion}

The primary end-point of the present study was to investigate the immunogenicity of mRNA-based COVID-19 vaccines, evaluated by measurement of serum anti-SARS-CoV-2 IgG NAb, in a large series of ASD patients. According to our data, a significantly higher percentage of patients failed to mount a response within 1-3 weeks after completion of the vaccination cycle when compared to controls, particularly for $\mathrm{CV}$ or systemic vasculitis. Consistently, serum levels of anti-SARS-CoV-2 IgG Nab were significantly lower in the whole ASD series and in different patients' subgroups. Interestingly, failure to mount an antibody response to COVID-19 vaccine correlated with coexistent ASD-related interstitial lung disease and some ongoing treatments, namely glucocorticoids, mycophenolate mofetil, and/or rituximab. With regard to the overall safety of both mRNA vaccines, no severe AEs or disease flares were observed in our ASD series; only mild and transitory AEs were recorded in comparable percentages among vaccine responder and nonresponder patients.

During the last few months, other observational studies on the same 
Table 3

Univariate logistic regression analysis using vaccine non-response as dependent variable.

\begin{tabular}{lll}
\hline & OR & P value \\
\hline Age & $0.990(0.973-1.008)$ & 0.283 \\
Female gender & $1.426(0.733-2.775)$ & 0.296 \\
ASD-related complications & & \\
Interstitial lung disease & $2.106(1.198-3.702)$ & $\mathbf{0 . 0 1 0}$ \\
Skin ulcers & $1.050(0.534-2.064)$ & 0.889 \\
Renal involvement & $1.942(0.618-6.098)$ & 0.256 \\
Pulmonary arterial hypertension & $2.469(0.858-7.110)$ & 0.094 \\
Gastrointestinal involvement & $0.768(0.173-3.405)$ & 0.728 \\
Treatment & & \\
Glucocorticoids & $2.27(1.321-3.903)$ & $\mathbf{0 . 0 0 3}$ \\
Methotrexate & $0.836(0.394-1.772)$ & 0.640 \\
Sulfasalazine & $2.215(0.227-21.631)$ & 0.494 \\
Hydroxychloroquine & $0.505(0.241-1.058)$ & 0.070 \\
Mycophenolate & $3.078(1.701-5.567)$ & $<\mathbf{0 . 0 0 0 1}$ \\
Azathioprine & $0.303(0.040-2.290)$ & 0.247 \\
TNF inhibitors & $0.288(0.038-2.176)$ & 0.228 \\
Abatacept & $0.653(0.082-5.192)$ & 0.687 \\
Rituximab & $6.739(2.956-15.367)$ & $<\mathbf{0 . 0 0 0 1}$ \\
IL-6 inhibitors & $0.319(0.042-2.416)$ & 0.268 \\
JAK inhibitors & $1.836(0.498-6.773)$ & 0.361 \\
Belimumab & $1.101(0.241-5.039)$ & 0.901 \\
\hline
\end{tabular}

Legend: ASD, autoimmune systemic diseases; IL-6, interleukin-6; JAK, Janus kinase; TNF, tumor necrosis factor.

topic have being published [13-16,19]. Only few of these studies investigated large series of patients with variegated diagnoses [14-16]; on the other hand, the remaining evidence is based on small case series focusing on single disorders such as SLE or RA [13,20-22].

The largest, controlled analysis was reported by Furer and coworkers that assessed the immunogenicity and safety of two-dose regimen BNT162b2 mRNA vaccine in adult with ASD [16]. The authors compared 686 patients with a smaller number of healthy controls $(\mathrm{n}=$ 121) and found a significantly reduced immunogenicity in the ASD cohort ( $86 \%$ vs $100 \%)$ [16].

Similar results ( $86 \%$ seroconversion) have been reported in a smaller, not controlled, cohort of ASD patients [15] while a higher immunogenic response (94\% seroconversion) was obtained in RA and SLE [14]. However, in the latter study the most likely explanation was a young age of the enrolled subjects [14]. Besides the evidences of lower seroconversion rate in patients with ASD compared to the general population, the titer of anti-SARS-CoV-2 IgG NAb was also reported to be lower in ASD patients compared to controls [13]. This observation was confirmed by studies on specific diseases such as SLE [23] and RA [20]. Finally, a markedly impaired serological response to the COVID-19 vaccine was reported in a cohort including 73 patients with RA and 61 patients with SLE [22]; the overall immunogenicity rate was $77 \%$, a lower figure compared to previous studies, probably due to the timing of the assessment which was performed only one week after the second dose of vaccine [22]. Fewer patients with RA $(49,67 \%)$ than those with SLE $(54,89 \%)$ had measurable antibodies against SARS- CoV- 2 [22].

The results of the present study, including homogeneous subgroups of ASD are in keeping with the main findings of the few available reports [22]. Although the possibility of a direct comparison is limited by the different study protocols and cohort composition, our findings further support the role of some risk factors for impaired vaccine immunogenicity, mainly treatment with mycophenolate mofetil [14-16] or rituximab [13-16,24]; the latter representing the main cause of non-response to anti-SARS-CoV-2 vaccine (39\% seropositivity) [14-16,24-26], as also suggested by the results of univariate analysis focusing on the factors associated with vaccine non-response. A recent review article underlined the role of anti-CD20 therapies in blunting the humoral immune response to vaccines, which could be counterbalanced by preserved T-cell response, as well as the need to develop personalized peri-vaccination management strategy $[5,24,25,27]$.

On the other hand, the prejudicial role of low-dose glucocorticoids [14, 16], methotrexate [14-16,21,22], and abatacept [16] on COVID-19 vaccine immunogenicity still remains controversial, while other molecules, such as belimumab, has been shown to don't interfere with antibody responses [22]. The role of increasing age in blunting immunogenicity of vaccines is suggested by only one monocentric cohort study [16]. Previous studies consistently report that, after the completion of the vaccination cycle, overall disease activity remained mostly stable $[13,15,16]$, while only a few cases of flares were reported $[15,16]$. De novo onset of arthritis was seen in a patient with interstitial lung disease and positive RF [15] and a fulminant hemorrhagic cutaneous vasculitis with subsequent fatal sepsis caused the death of a patient with ANCA-associated vasculitis [16]. The highest percentage $(6.5 \%)$ of post-vaccination flare was described by an Italian study focusing on RA cases [28]. Pre-vaccine discontinuation of the anti-rheumatic therapies in this population of patients may account for the discrepancy with other reports. In our series, disease reactivation was rare and overall, not alarming. Of note, the majority of patients experiencing flares had cryoglobulinemic vasculitis (60\%), a condition not adequately evaluated by previous studies [15] or included, as anecdotal cases, in a miscellanea of ASD patients $[13,16]$.

Although the blunted humoral response to COVID-19 vaccination is a sufficient reason of concern, the complete lack of NAb response represents the most crucial consequence of the immunological derangement characterizing ASD patients, along with few immune-modifier drugs such as rituximab. Despite some well-identified iatrogenic risk factors, the nonresponse observed in over $13 \%$ of ASD patients (25\% in CV) remains largely unpredictable; therefore, it becomes crucial to early identify NAb negative individuals. Another compelling open question is the management of patients who show inadequate or absent humoral response. These seronegative patients, presumed to be immunized against SARS-CoV-2, may be at high risk of symptomatic infection. Although a percentage of seronegatives may develop a delayed seroconversion, around $23 \%$ as suggested by a recent observational study [29], this does not affect the significant difference between ASD patients and controls in the prevalence of non-responders. Consequently, in our opinion, the early measurement of serum NAb titer after completion of the vaccination cycle should be included in the routine assessment of all immunocompromised patients; this information may guide the subsequent management in terms of both timing and type of vaccine to be administered as a booster-dose, as well as the concurrent immune-modifier treatments. Indeed, in an early report, a booster-dose of vaccine in patients with ASD seems able to induce an amplified antibody response in the majority of subjects [30,31].

A proper management strategy for ASD patients during pre- and postvaccination cycle should take in account the possible immunosuppressive effect of different treatments; the latter include rituximab and mycophenolate mofetil, mainly responsible for impaired immunogenicity, and some commonly used drugs such as corticosteroids. Therefore, the production of adequate therapeutic guidelines on the basis of recently available real-life experiences is eagerly awaited. The determination of NAb serum levels within the first 3 weeks from the second dose of vaccine would allow the identification of essentially three groups of patients: full responders, patients with suboptimal response, nonresponders. In our opinion, patients showing a suboptimal response should be prioritized for a booster-dose of vaccine, while the administration of a different type of vaccine (for example from mRNA to vectorbased vaccine) could be attempted in non-responder individuals. In any case, a careful evaluation of individual patients can model a personalized strategy, such as the tapering/discontinuation or delayed 
therapeutic sessions of immunosuppressive treatments, mainly for rituximab, before a booster-dose of vaccine.

\section{Conclusion}

The ongoing pandemic still represents a complex challenge for the management of frail patients. A continuous adjustment of management strategies is still required, considering the evolution of pandemic, the clinic-pathogenetic peculiarities of different patients' settings, and their unpredictable response to vaccination. Hopefully, a widespread availability of serological testing may allow to recognize patients previously infected by SARS-CoV-2; rather it may be decisive to early identify patients with impaired/absent seroconversion needing an individualized therapeutic strategy.

\section{Authors' statements}

Clodoveo Ferri: conceptualisation, study design, supervision, writing, investigation, methodology, project administration, literature search; Francesco Ursini: formal analysis, figures, data interpretation, writing; Laura Gragnani: literature search, writing, methodology; Vincenzo Raimondo: data collection; Dilia Giuggioli: data collection; Rosario Foti: data collection; Maurizio Caminiti: data collection; Domenico Olivo: data collection; Giovanna Cuomo: data collection; Marcellla Visentini: data collection; Fabio Cacciapaglia: data collection; Roberta Pellegrini: data collection; Erika Pigatto: data collection; Teresa Urraro: data collection; Caterina Naclerio: data collection; Antonio Tavoni: data collection; Lorenzo Puccetti: data collection; Giuseppe Varcasia: data collection; Ilaria Cavazzana: data collection; Massimo L'Andolina: data collection; Piero Ruscitti: data collection; Marta Vadacca: data collection; Pietro Gigliotti: data collection; Francesca La Gualana: data collection; Franco Cozzi: data collection; Amelia Spinella: data collection; Elisa Visalli: data collection; Ylenia Dal Bosco: data collection; Giorgio Amato: data collection; Francesco Masini: data collection; Giuseppa Pagano Mariano: data collection; Raffaele Brittelli: data collection; Vincenzo Aiello: data collection; Rodolfo Caminiti: data collection; Daniela Scorpiniti: data collection; Giovanni Rechichi: data collection; Tommaso Ferrari: data collection; Monica Monti: data collection; Giusy Elia: data collection; Franco Franceschini: data collection; Riccardo Meliconi: data collection; Milvia Casato:data collection; Florenzo Iannone data collection; Roberto Giacomelli: data collection; Poupak Fallahi: data collection; Stefano Angelo Santini: methodology; Anna Linda Zignego: data collection; Alessandro Antonelli: conceptualisation, writing. All authors critically revised the paper and approved the submitted version.

\section{Funding}

This research did not receive any specific grant from funding agencies in the public, commercial, or not-for-profit sectors.

\section{Data availability}

Data will be made available on request.

\section{Acknowledgements}

The authors would like to thank Veronica Magistri (Application Specialist, Abbott Diagnostics Rome, Italy) for her kind technical support.

\section{References}

[1] J.L. Pablos, M. Galindo, L. Carmona, A. Lledó, M. Retuerto, R. Blanco, et al., Clinical outcomes of hospitalised patients with COVID-19 and chronic inflammatory and autoimmune rheumatic diseases: a multicentric matched cohort study, Ann. Rheum. Dis. 79 (2020) 1544-1549.

[2] K.M. D'Silva, A. Jorge, A. Cohen, N. McCormick, Y. Zhang, Z.S. Wallace, et al., COVID-19 outcomes in patients with systemic autoimmune rheumatic diseases compared to the general population: a US multicenter, comparative cohort study, Arthritis Rheum. 73 (2021) 914-920.

[3] C. Ferri, D. Giuggioli, V. Raimondo, M. L'Andolina, A. Tavoni, R. Cecchetti, et al., COVID-19 and rheumatic autoimmune systemic diseases: report of a large Italian patients series, Clin. Rheumatol. 39 (2020) 3195-3204.

[4] A. Antonelli, P. Fallahi, G. Elia, F. Ragusa, S.R. Paparo, V. Mazzi, et al., Effect of the COVID-19 pandemic on patients with systemic rheumatic diseases, Lancet Rheumatol 3 (2021) e675-e676.

[5] F. Fagni, D. Simon, K. Tascilar, V. Schoenau, M. Sticherling, M.F. Neurath, et al., COVID-19 and immune-mediated inflammatory diseases: effect of disease and treatment on COVID-19 outcomes and vaccine responses, Lancet Rheumatol 3 (2021) e724-e736.

[6] C. Ferri, D. Giuggioli, V. Raimondo, M. L’Andolina, L. Dagna, A. Tavoni, et al., Covid-19 and rheumatic autoimmune systemic diseases: role of pre-existing lung involvement and ongoing treatments, Curr. Pharmaceut. Des. (2021).

[7] D.D. Freites Nuñez, L. Leon, A. Mucientes, L. Rodriguez-Rodriguez, J. Font Urgelles, A. Madrid García, et al., Risk factors for hospital admissions related to COVID-19 in patients with autoimmune inflammatory rheumatic diseases, Ann. Rheum. Dis. 79 (2020) 1393-1399.

[8] C. Ferri, D. Giuggioli, V. Raimondo, L. Dagna, V. Riccieri, E. Zanatta, et al., COVID19 and systemic sclerosis: clinicopathological implications from Italian nationwide survey study, Lancet Rheumatol 3 (2021) e166-e168.

[9] M. Gianfrancesco, K.L. Hyrich, S. Al-Adely, L. Carmona, M.I. Danila, L. Gossec, et al., Characteristics associated with hospitalisation for COVID-19 in people with rheumatic disease: data from the COVID-19 Global Rheumatology Alliance physician-reported registry, Ann. Rheum. Dis. 79 (2020) 859-866.

[10] F. contributors, Severity of COVID-19 and survival in patients with rheumatic and inflammatory diseases: data from the French RMD COVID-19 cohort of 694 patients, Ann. Rheum. Dis. (2020).

[11] R. Hasseli, U. Mueller-Ladner, B.F. Hoyer, A. Krause, H.M. Lorenz, A. Pfeil, et al., Older age, comorbidity, glucocorticoid use and disease activity are risk factors for COVID-19 hospitalisation in patients with inflammatory rheumatic and musculoskeletal diseases, RMD Open 7 (2021).

[12] R. D'Amelio, R. Asero, M.A. Cassatella, B. Laganà, C. Lunardi, P. Migliorini, et al., Anti-COVID-19 vaccination in patients with autoimmune-autoinflammatory disorders and primary/secondary immunodeficiencies: the position of the task force on behalf of the Italian immunological societies, Biomedicines 9 (2021).

[13] U.M. Geisen, D.K. Berner, F. Tran, M. Sümbül, L. Vullriede, M. Ciripoi, et al., Immunogenicity and safety of anti-SARS-CoV-2 mRNA vaccines in patients with chronic inflammatory conditions and immunosuppressive therapy in a monocentric cohort, Ann. Rheum. Dis. 80 (2021) 1306-1311.

[14] J.A. Ruddy, C.M. Connolly, B.J. Boyarsky, W.A. Werbel, L. Christopher-Stine, J. Garonzik-Wang, et al., High antibody response to two-dose SARS-CoV-2 messenger RNA vaccination in patients with rheumatic and musculoskeletal diseases, Ann. Rheum. Dis. 80 (2021) 1351-1352.

[15] Y. Braun-Moscovici, M. Kaplan, M. Braun, D. Markovits, S. Giryes, K. Toledano, et al., Disease activity and humoral response in patients with inflammatory rheumatic diseases after two doses of the Pfizer mRNA vaccine against SARS-CoV-2, Ann. Rheum. Dis. 80 (2021) 1317-1321.

[16] V. Furer, T. Eviatar, D. Zisman, H. Peleg, D. Paran, D. Levartovsky, et al., Immunogenicity and safety of the BNT162b2 mRNA COVID-19 vaccine in adult patients with autoimmune inflammatory rheumatic diseases and in the general population: a multicentre study, Ann. Rheum. Dis. 80 (2021) 1330-1338.

[17] B.J. Boyarsky, J.A. Ruddy, C.M. Connolly, M.T. Ou, W.A. Werbel, J.M. GaronzikWang, et al., Antibody response to a single dose of SARS-CoV-2 mRNA vaccine in patients with rheumatic and musculoskeletal diseases, Ann. Rheum. Dis. (2021).

[18] J.W. Bijlsma (Ed.), EULAR Compendium on Rheumatic Diseases, New Edition ed., BMJ Publishing Group Ltd, London, 2018.

[19] L. Boekel, M. Steenhuis, F. Hooijberg, Y.R. Besten, Z.L.E. van Kempen, L. Y. Kummer, et al., Antibody development after COVID-19 vaccination in patients with autoimmune diseases in The Netherlands: a substudy of data from two prospective cohort studies, Lancet Rheumatol (2021).

[20] A. Rubbert-Roth, N. Vuilleumier, B. Ludewig, K. Schmiedeberg, C. Haller, J. von Kempis, Anti-SARS-CoV-2 mRNA vaccine in patients with rheumatoid arthritis, Lancet Rheumatol 3 (2021) e470-e472.

[21] C.M. Connolly, B.J. Boyarsky, J.A. Ruddy, W.A. Werbel, L. Christopher-Stine, J. M. Garonzik-Wang, et al., Absence of humoral response after two-dose SARS-CoV-2 messenger RNA vaccination in patients with rheumatic and musculoskeletal diseases: a case series, Ann. Intern. Med. 174 (2021) 1332-1334.

[22] C. Ammitzbøll, L.E. Bartels, J. Bøgh Andersen, S. Risbøl Vils, C. Elbaek Mistegård, A. Dahl Johannsen, et al., Impaired antibody response to the BNT162b2 messenger RNA coronavirus disease 2019 vaccine in patients with systemic lupus erythematosus and rheumatoid arthritis, ACR Open Rheumatol 3 (2021) 622-628. 
[23] P.M. Izmirly, M.Y. Kim, M. Samanovic, R. Fernandez-Ruiz, S. Ohana, K. K. Deonaraine, et al., Evaluation of Immune Response and Disease Status in SLE Patients Following SARS-CoV-2 Vaccination, Arthritis Rheumatol, 2021.

[24] M.M. Bonelli, D. Mrak, T. Perkmann, H. Haslacher, D. Aletaha, SARS-CoV-2 vaccination in rituximab-treated patients: evidence for impaired humoral but inducible cellular immune response, Ann. Rheum. Dis. 80 (2021) 1355-1356.

[25] R. Spiera, S. Jinich, D. Jannat-Khah, Rituximab, but not other antirheumatic therapies, is associated with impaired serological response to SARS- CoV-2 vaccination in patients with rheumatic diseases, Ann. Rheum. Dis. 80 (2021) 1357-1359.

[26] M.B. Moor, F. Suter-Riniker, M.P. Horn, D. Aeberli, J. Amsler, B. Möller, et al., Humoral and cellular responses to mRNA vaccines against SARS-CoV-2 in patients with a history of CD20 B-cell-depleting therapy (RituxiVac): an investigatorinitiated, single-centre, open-label study, Lancet Rheumatol (2021).

[27] D. Simon, K. Tascilar, K. Schmidt, B. Manger, L. Weckwerth, M. Sokolova, et al., Brief Report: Humoral and Cellular Immune Responses to SARS-CoV-2 Infection and Vaccination in B Cell Depleted Autoimmune Patients, Arthritis Rheumatol, 2021.

[28] R. Bixio, D. Bertelle, M. Masia, F. Pistillo, A. Carletto, M. Rossini, Incidence of Disease Flare after BNT162b2 Coronavirus Disease 2019 Vaccination in Patients with Rheumatoid Arthritis in Remission, ACR Open Rheumatol, 2021.

[29] S. Frey, C.M. Connolly, T.P. Chiang, M. Teles, J.L. Alejo, B.J. Boyarsky, et al., Antibody kinetics in patients with rheumatic diseases after SARS-CoV-2 mRNA vaccination, Lancet Rheumatol 3 (2021) e753-e754.

[30] C.M. Connolly, M. Teles, S. Frey, B.J. Boyarsky, J.L. Alejo, W.A. Werbel, et al. Booster-dose SARS-CoV-2 vaccination in patients with autoimmune disease: a case series, Ann. Rheum. Dis. (2021).

[31] F.N. Albach, G.R. Burmester, R. Biesen, Successful BNT162b2 booster vaccinations in a patient with rheumatoid arthritis and initially negative antibody response, Ann. Rheum. Dis. 80 (2021) 1361-1362. 